\title{
An intermediate water Cherenkov detector for the Hyper-Kamiokande experiment: overview and status
}

\author{
Evangelia Drakopoulou*t \\ University of Edinburgh \\ E-mail: Evangelia.Drakopoulou@ed.ac.uk
}

The proposed Hyper-Kamiokande (Hyper-K) is a next generation underground large water Cherenkov (WCh) detector. Hyper-K will act as a far detector to measure the oscillated neutrino flux from the long-baseline neutrino experiment using $0.6 \mathrm{GeV}$ neutrinos produced by a 1.3 MW proton beam at J-PARC in Japan. It has a broad program of physics and astrophysics mainly focusing on the precise measurement of the lepton mixing matrix and the leptonic CP asymmetry. The main systematic uncertainties on these measurements arise from uncertainties on the modelling of neutrino-nucleus interactions. To minimise these uncertainties an intermediate WCh detector, E61, has been proposed. E61 aims to investigate the relation between the neutrino energy and the lepton kinematics using measurements of final state muons at many different off-axis angles. The detector will use a new technique to enhance its performance, neutron tagging. The water in the tank will be doped with Gd that allows to tag neutrons. Tagging events by the presence and number of final-state neutrons provides a unique capability to discriminate between different (anti)neutrino interactions. A neutron multiplicity spectrum will be measured aiming to improve the current knowledge of neutrino nucleon interactions and thus help in the background reduction as well in the systematic uncertainty reduction at Hyper-K. We will discuss the expected physics reach and the status of the E61 experiment focusing on the benefits from the off-axis spanning and neutron tagging techniques.

35th International Cosmic Ray Conference - ICRC2017

10-20 July, 2017

Bexco, Busan, Korea

* Speaker.

${ }^{\dagger}$ for the J-PARC E61 collaboration. 


\section{Introduction}

The uncertainties on the modelling of neutrino interactions are one the largest sources of uncertainty for the next generation neutrino experiment, Hyper-K. To minimise this uncertainty the E61 detector located at $1 \mathrm{~km}$ baseline is proposed. In section 2, the Hyper-K experiment is described. In section 3, we give an overview of the E61 experiment and the current status. In sections 4, 5 and 6 we discuss how the neutron multiplicity measurements of the E61 detector can enhance the studies of atmospheric neutrinos, proton decay and supernova neutrinos in Hyper-K. Conclusions and future developments are included in section 7.

\section{The Hyper Kamiokande experiment}

Hyper-K [1] aims for an observation of $\mathrm{CP}$ violation in neutrino oscillations and precise measurements of other oscillation parameters, such as the mixing angle $\theta_{23}$ and $\Delta m_{23}^{2}$. Based on the highly successful Super-Kamiokande (Super-K) detector [2], the Hyper-K detector performance will be further enhanced by an order of magnitude larger fiducial mass and higher performance photodetectors thus constituting a powerful extension of the Japan-based neutrino program. The design of Hyper-K calls for the staged construction of two cylindrical tanks with a total (fiducial) mass of 0.52 (0.37) Mton, making it 10 (17) times larger than its predecessor [3]. Figure 1 shows the design of the first planned tank. The first tank will be located near the current Super-K site in the Tochibora mine. The possibility to locate the second tank in South Korea to further improve the sensitivity for determining the neutrino mass ordering and leptonic $\mathrm{CP}$ asymmetry measurements is being investigated [4]. A detailed overview of the Hyper-K experiment is discussed in a separate contribution to these proceedings [5].

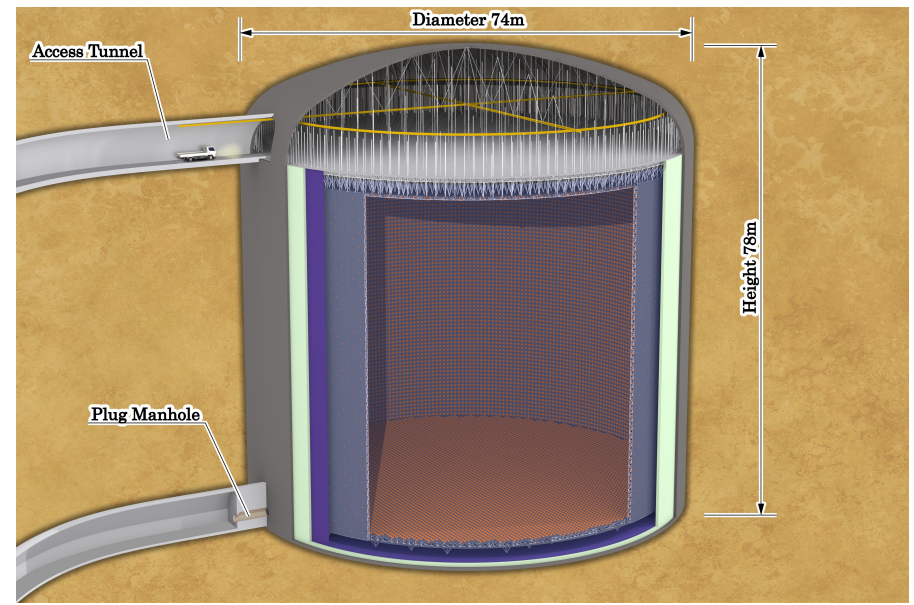

Figure 1: The Hyper-Kamiokande experiment and infrastructure.

\section{The E61 experiment}

Neutrino interaction model uncertainties are the main systematics uncertainties for the near and far detector analyses in neutrino oscillation experiments. Many of the uncertainties on the 
modelling of neutrino interactions arise from nuclear effects and these can be minimised with an intermediate detector that uses the same nuclear target (water) as the far detector. A main aim of the E61 detector is to provide the first direct experimental constraint on the relationship between lepton kinematics and neutrino energy using measurements of final state muons at many different off-axis angles. E61 will take advantage of the energy dependence of the neutrino flux with offaxis angle by spanning a continuous range of 1 to 4 degrees in off-axis angle. This technique can significantly reduce uncertainties from neutrino interaction modelling in oscillation analyses [6]. Moreover, to enhance the performance of the detector, the water in the tank will be doped with Gadolinium allowing the discrimination of neutrinos and antineutrinos and hence discriminating between signal and background events. A measurement of the neutron multiplicity will also be performed to be used by Hyper-K. A new intermediate detector collaboration, E61, has recently been formed merging the previous NuPRISM [6] and TITUS [7] proposals.

\subsection{Off-axis spanning}

The E61 detector design will be similar to the baseline described in the NuPRISM proposal [6] and it will be doped with Gd to allow neutrino-antineutrino separation and distinguish between different interaction types as described in the TITUS proposal [7]. The detector consists of a frame of $8 \mathrm{~m}$ diameter and $10 \mathrm{~m}$ height instrumented with photomultipliers (PMTs) that moves vertically within a $50 \mathrm{~m}$-tall water tank (with a diameter of $10 \mathrm{~m}$ ) as it is shown in Figure 2. In the same Figure, the neutrino energy spectra at several different off-axis angles in the neutrino beam line is shown. As the detector is moved to larger off-axis angles relative to the beam direction, the peak energy of the neutrino energy spectrum is lowered and the size of the high-energy tail is reduced.

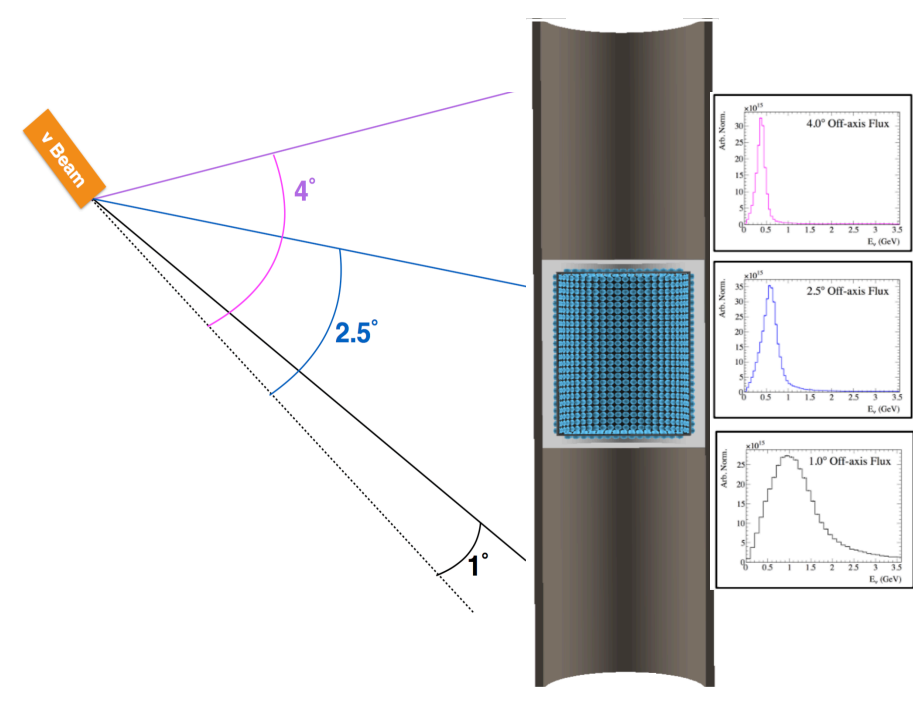

Figure 2: The E61 detector.

The off-axis angle for a single neutrino interaction can be determined from the reconstructed vertex position. In this way, the detector can be logically divided into slices of off-axis angles based on the reconstructed vertex of each event. The muon momentum and angle relative to the mean neutrino direction can be measured for each slice of off-axis angle. Linear combinations of the measurements in each slice can be used to produce an effective muon momentum and angle 
distribution for a Gaussian-like beam at energies between 0.4 and $1.2 \mathrm{GeV}$. On-axis measurements can be used to subtract the high energy tail and further off-axis measurements to subtract the low energy tail resulting in a "pseudo-mono-chromatic" neutrino energy spectra. Using "pseudo-monochromatic" beams this detector has the ability to measure the true neutrino energy dependence of both charged and neutral current interactions. These measurements are expected to significantly enhance the reach of oscillation experiments, since the energy dependence of signal and background processes must be understood in order to place strong constraints on oscillation parameters.

By producing a wide variety of muon momentum and angle distributions a linear combination method can be used to predict the observed muon kinematic distributions for the oscillated flux. This is shown in Figure 3. This linear combination method can use a wide variety of neutrino flux shapes between 0.4 and $1.0 \mathrm{GeV}$ to predict the reconstructed energy distribution at the far detector (Hyper-K or Super-K). Being able to reproduce all possible spectra after neutrino oscillations in the far detector will allow to significantly reduce many of the uncertainties associated with the modelling of neutrino-nucleus interactions. An example of the use of linear combination of offaxis fluxes to predict the oscillated spectrum in the Super-K detector is shown in Figure 3. A good agreement has been achieved, except at low neutrino energies (below $400 \mathrm{MeV}$ ). This is due to the fact that the maximum off-axis angle of $4^{\circ}$ leads to a spectrum with peak at $380 \mathrm{MeV}$ making it difficult to reproduce an arbitrary flux shape at lower energies. With this approach the systematic error due to neutrino interaction model uncertainties is reduced to $1 \%$ compared to $4 \%$ that was estimated by previous experiments.

The E61 experiment can perform high-precision measurements of neutrino cross sections on water, including electron neutrino measurements and the first measurements of neutral current interactions as a function of neutrino energy. In addition, this detector will search for sterile neutrino oscillations with multiple energy spectra.

\subsection{Neutron Tagging}

To further enhance the detector performance, the E61 collaboration plans to also use another technique. The water in the tank will be doped with $\mathrm{Gd}$, which has a very high cross section for neutron capture (49700 barns for natural Gd compared to 0.19 millbarns for oxygen). These captures produce $8.6 \mathrm{MeV}$ of energy in one or more photons. In WCh detectors these photons produce electrons by Compton scattering and pair production, and they in turn produce Cherenkov photons that can be detected in the photodetectors, thus permitting the tagging of neutrons.

Charged current quasi-elastic (CCQE) scattering of a neutrino (antineutrino) on a neutron (proton) produces a negative (positive) charged lepton and a proton (neutron). Tagging neutrons in the final state identifies antineutrinos. This provides charge separation information particularly in situations where the lepton charge is not known, as in the case of a WCh detector. The neutron tagging technique can discriminate between CCQE and non-CCQE neutrino interactions, which typically produce more neutrons. The ability to discriminate between CCQE and non-CCQE neutrino interactions in a statistical approach can enhance the purity of the selected CCQE events and result in better signal-background separation in the E61 experiment. The neutrino energy reconstruction assumes CCQE interactions of neutrinos. Enhancing the purity of the CCQE event sample will thus improve the neutrino energy reconstruction, which is strongly correlated to the fraction of un-detected energy from hadron production. Neutron tagging will improve our understanding 


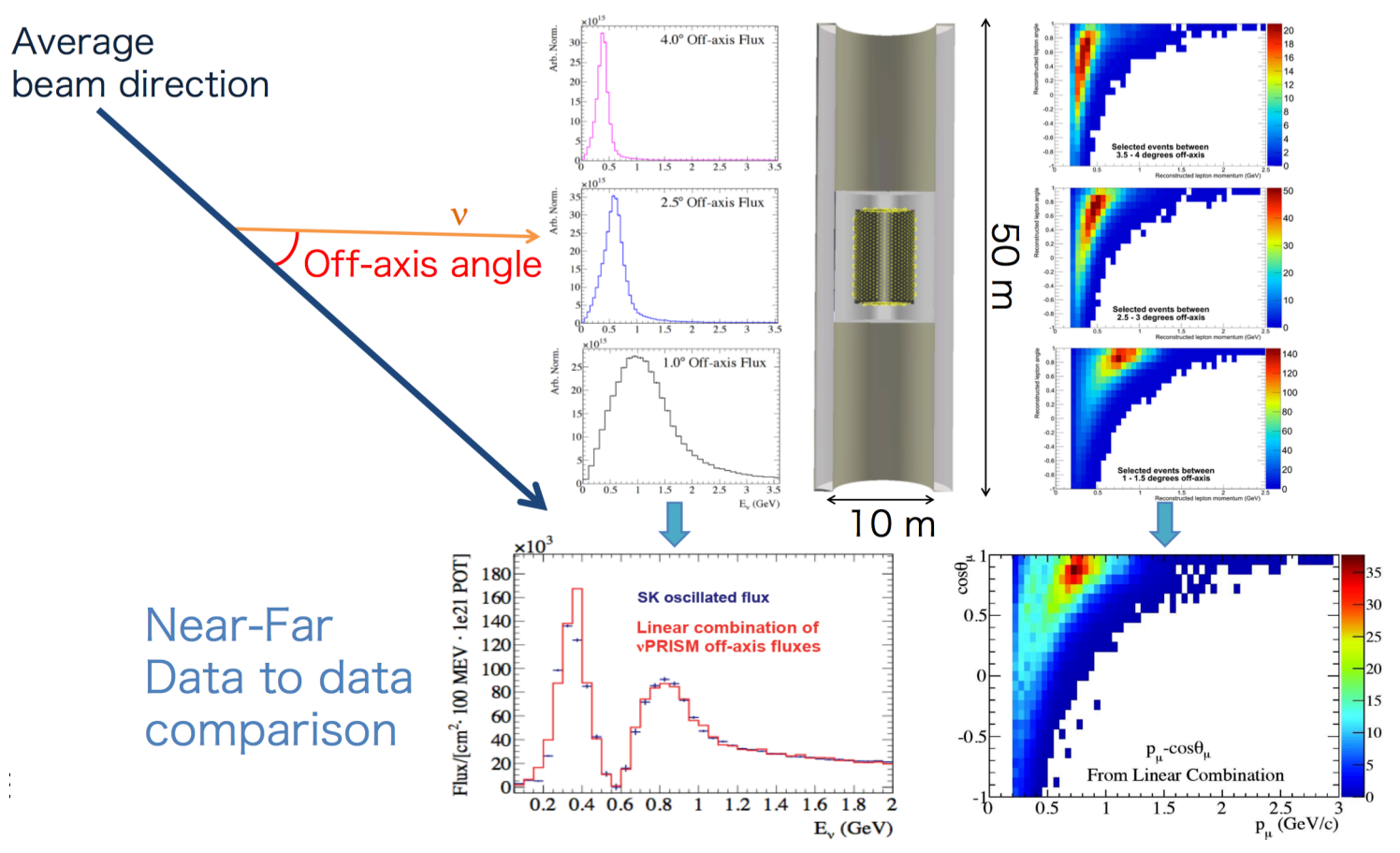

Figure 3: The use of a linear combination method in the E61 detector. A linear combination of off-axis fluxes is used to reproduce the oscillated flux at the far (here the Super-K) detector. Linear combination of different muon momentum and angle distributions predicts the muon kinematic distributions for the oscillated flux.

of nuclear effects in neutrino interactions, thus reducing the large uncertainties that exist within the different neutrino interaction models due to final state interactions. The direct measurement of the neutron multiplicity in an intermediate WCh detector with the same target medium would significantly reduce these background uncertainties. Minimising these uncertainties will lead to a reduction of the systematic uncertainties in neutrino experiments, which is vital for the precise measurement of the leptonic CP asymmetry.

The feasibility of Gd doping in a WCh detector has already been successfully tested by the Evaluating Gadolinium Action on Detector System (EGADS) [8] collaboration and led to the decision to dope the Super-K detector with Gd (about $0.1 \%$ of Gd), which is scheduled for 2018. The E61 collaboration is currently performing studies to address the challenges of the neutron multiplicity measurement. These studies include the ability to identify the tagged neutrons with the correct primary vertex and to reject neutrons from background sources such as those induced by beam interactions in the sand downstream of the detector, or by cosmic rays. Several improvements have been recently performed in the simulation and reconstruction tools allowing to estimate the event rates of neutrons from beam interactions in the detector, beam interactions in the sand surrounding the detector and from cosmic ray interactions and also of radioactivity in the PMTs that 
could mimic the low energy events from neutron captures. These studies along with further analyses will be used to investigate the necessary thickness of an outer detector region which will capture entering neutrons and will be used as a veto.

\subsection{Detector Construction}

The detector will be constructed in two phases. In Phase- 0 a smaller detector of $6 \mathrm{~m}$ tall and $8 \mathrm{~m}$ diameter will be located on the surface at approximately $300 \mathrm{~m}$ baseline in the J-PARC neutrino beam. This detector will act as a prototype to confirm the detector performance. Construction is foreseen to begin during 2018 aiming to take first data in 2021. It will perform precision measurement (at the precision of $3 \%$ for $\mathrm{E}_{v}<1 \mathrm{GeV}$ ) of neutrino cross sections and study neutron multiplicities in neutrino interactions. The E61 collaboration plans to use multi-PMT modules hosting 3-inch PMTs to enhance the granularity in imaging Cherenkov rings compared to larger PMTs and profit from the better timing resolution and improved directional information of this design. A drawing of the multi-PMT design is shown in Figure 4. This design allows to integrate the photomultipliers of the inner and outer detector in a single unit facilitating the implementation. It is based on the multi-PMT module used by the KM3NeT collaboration [9] modified for the specific needs of the E61 experiment. Having established the technology and detector performance, the Phase-1 detector construction will begin in 2021 aiming to a fully operational detector by 2024 .

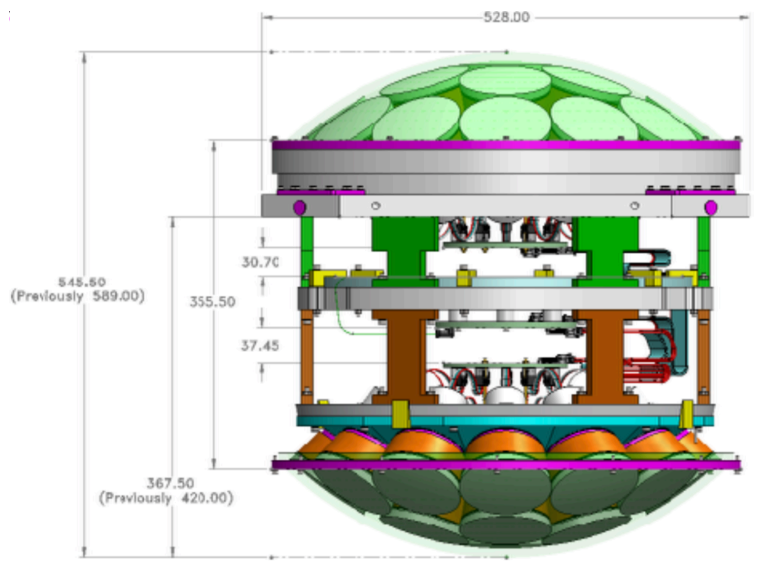

Figure 4: A drawing of the multi-PMT module hosting 3-inch PMTs. The upper (lower) hemisphere will cover the inner (outer) detector region.

\section{Atmospheric neutrinos at Hyper-K}

Atmospheric neutrinos provide an independent and complementary information to the accelerator beam program on the study of neutrino oscillations with the Hyper-K WCh detector. Atmospheric neutrinos passing through the Earth will experience the matter effect thus affecting neutrino oscillations between different types. The extent of these effects depends upon the mass hierarchy and Hyper-K with its large volume will have a good sensitivity (of more than $3 \sigma$ after 5 years, more than $5 \sigma$ after 10 years ) to the mass hierarchy. Combining the complementary measurements 
from both accelerator and atmospheric neutrinos Hyper-K can enhance its physics capability [1], [3].

Neutron tagging in the E61 experiment will improve our knowledge on neutron multiplicities in neutrino-nucleus interactions providing useful information to existing theoretical models. These studies will allow Hyper-K to reduce the errors in atmospheric neutrino measurements.

\section{Proton decay at Hyper-K}

Super-K has set the lowest limit of proton lifetime. Being approximately 10 times larger than Super-K, the Hyper-K detector will be able to collect even larger numbers of protons and overtake the current reach by Super-K within two years. Hyper-K will increase the existing sensitivity to proton decay by an order of magnitude being also able to examine most of Grand Unified Theory models [1], [3]. Proton decay analysis uses neutron tagging to enhance proton decay searches. Tagging neutrons allows to reject background events by requiring signal without neutron candidate events in the final state. This can separate signal from background in proton decay final states reducing the main background to proton decays in $\mathrm{e}^{+} \pi^{0}$ coming from atmospheric neutrinos.

The Gd-doped E61 detector has a small volume to allow for proton decay searches. The detector will measure the neutron multiplicities in neutrino-nucleus interactions providing useful information to the theoretical models. Reducing modelling uncertainties will allow Super-K and Hyper-K to reduce the errors on the expected number of neutrons improving their detection ability in proton decay searches. Measurements of the neutron content in the cross section events performed by E61 can thus provide an important input to the background determination for proton decays [7].

\section{Supernova neutrinos at Hyper-K}

Core collapse supernova explosions are the final evolutionary stage of massive stars (with masses more than eight times the mass of the Sun). Such explosions release $99 \%$ of their energy in neutrinos. The Kamiokande WCh detector, the ancestor of Super-Kamiokande, firstly observed neutrinos from Supernova 1987A [10]. Supernova neutrinos can provide information about the formation of the neutron star and allow to investigate the detailed mechanisms of supernova explosions. Neutron tagging can be used in the detection of diffuse supernova neutrino background. In particular, tagging neutrons can allow to separate genuine neutrinos from various radiogenic and spallation backgrounds. In the event of a core collapse supernova, the detection of neutrons can be used to help discriminate among different interactions in the water such as inverse beta decay and neutrino-oxygen scattering [7].

Given its small volume, the E61 detector will not observe neutrinos from supernova. The E61 detector will provide a good measurement of the expected number of neutrons from neutrinosnucleus interactions allowing for Super-K and Hyper-K experiments to improve their signal-background separation and enhance their detection ability. 


\section{Conclusions}

The E61 collaboration (merging the NuPRISM and TITUS collaborations) aims to construct an intermediate WCh detector in the baseline of the J-PARC neutrino beam. The water in the tank will be doped with $\mathrm{Gd}$ that allows to tag final state neutrons. Taking advantage of the neutron tagging technique and of the detector ability to measure the neutrino spectrum at different off-axis angles the E61 experiment aims to minimise systematic uncertainties arising from neutrino-nucleus interactions in Hyper-K. The study of the neutron component of the neutrino-nucleon interactions at given neutrino energies can be provided to Hyper-K. This can help in reducing the errors and backgrounds in beam, atmospheric, supernova neutrino and proton decay searches at Hyper-K.

\section{References}

[1] K. Abe et al, Physics potential of a long-baseline neutrino oscillation experiment using a J-PARC neutrino beam and Hyper-Kamiokande, Prog. Theor. Exp. Phys. (2015) $053 \mathrm{C} 02$ [c].

[2] Y. Ashie et al., Evidence for an oscillatory signature in atmospheric neutrino oscillation, Phys.Rev.Lett. 93 (2004) 101801 [hep-ex/ 0404034 ].

[3] K. Abe et al., Hyper-Kamiokande Design Report, [KEK-Preprint-2016-21, ICRR-Report-701-2016-1].

[4] K. Abe et al, Physics Potentials with the Second Hyper-Kamiokande Detector in Korea, (2016) [arXiv: 1611.06118].

[5] See contribution by S. Zsoldos in these proceedings.

[6] S. Bhadra et al, Letter of Intent to Construct a nuPRISM Detector in the J-PARC Neutrino Beamline, (2014) [arXiv: 1412.3086].

[7] C. Andreopoulos et al, TITUS: the Tokai Intermediate Tank for the Unoscillated Spectrum, (2016) [arXiv: 1606.08114 ].

[8] C. Xu for the Super-Kamiokande Collaboration, Current status of SK-Gd project and EGADS, J. Phys. Conf. Ser. 718 no.6, 5 (2016).

[9] KM3NeT Collaboration, Deep sea tests of a prototype of the KM3NeT digital optical module, Eur. Phys. J. C 74 (2014) 3056.

[10] K. Hirata et al., Observation of a neutrino burst from the supernova SN1987A, Phys. Rev. Lett. 58, (1987) 1490-1493. 